\title{
De novo, systemic, deleterious amino acid substitutions are common in large cytoskeleton-related protein coding regions
}

\author{
REBECCA J. STOLL ${ }^{1}$, GRACE R. THOMPSON ${ }^{1}$, MOHAMMAD D. SAMY ${ }^{1}$ and GEORGE BLANCK ${ }^{1,2}$ \\ ${ }^{1}$ Department of Molecular Medicine, Morsani College of Medicine, University of South Florida; \\ ${ }^{2}$ Immunology Program, H. Lee Moffitt Cancer Center and Research Institute, Tampa, FL 33612, USA
}

Received June 13, 2016; Accepted October 31, 2016

DOI: 10.3892/br.2016.826

\begin{abstract}
Human mutagenesis is largely random, thus large coding regions, simply on the basis of probability, represent relatively large mutagenesis targets. Thus, we considered the possibility that large cytoskeletal-protein related coding regions (CPCRs), including extra-cellular matrix (ECM) coding regions, would have systemic nucleotide variants that are not present in common SNP databases. Presumably, such variants arose recently in development or in recent, preceding generations. Using matched breast cancer and blood-derived normal datasets from the cancer genome atlas, CPCR single nucleotide variants (SNVs) not present in the All SNPs(142) or 1000 Genomes databases were identified. Using the Protein Variation Effect Analyzer internet-based tool, it was discovered that apparent, systemic mutations (not shared among others in the analysis group) in the CPCRs, represented numerous deleterious amino acid substitutions. However, no such deleterious variants were identified among the (cancer blood-matched) variants shared by other members of the analysis group. These data indicate that private SNVs, which potentially have a medical consequence, occur de novo with significant frequency in the larger, human coding regions that collectively impact the cytoskeleton and ECM.
\end{abstract}

Correspondence to: Dr George Blanck, Department of Molecular Medicine, Morsani College of Medicine, University of South Florida, 12901 Bruce B. Downs Boulevard, MDC7, Tampa, FL 33612, USA E-mail: gblanck@health.usf.edu

Abbreviations: CPCR, cytoskeleton-related protein coding regions; ECM, extracellular matrix; BRCA, breast cancer; TCGA, The Cancer Genome Atlas; SNV, single nucleotide variant; PROVEAN, Protein Variation Effect Analyzer; WXS, whole exome sequence; SNP, single nucleotide polymorphism; SOM, supporting online material

Key words: cytoskeleton-related protein coding regions, The Cancer Genome Atlas, breast cancer, genetic testing, systemic mutations, single nucleotide variants, private variants

\section{Introduction}

Genetic damage is largely random and therefore tends to affect the larger, functional regions of the human genome more frequently than the smaller regions (1). For example, a systematic study has revealed that cancer fusion genes, on average, are statistically, significantly larger than other human genes $(2,3)$. The large introns of potential cancer fusion genes presumably allow for many different productive recombination opportunities, i.e., many recombinations that would allow for exon juxtaposition and the generation of hybrid proteins. Smaller cancer fusion genes tend to be associated with the rare types of cancer, for example EWS RNA binding protein 1 in Ewing's sarcoma.

Cytoskeleton-related protein coding regions (CPCRs), including extracellular matrix (ECM) proteins, are among the largest coding regions in the human genome and are heavily mutated in various types of cancer $(1,4,5)$. The possibility that these coding regions would also be commonly vulnerable to de novo mutations, or mutations occurring in relatively recent past-generations, was considered, and therefore had not been included in the conventional single nucleotide polymorphism (SNP) databases. In the present study, CPCR single nucleotide variants (SNVs) that appeared in The Cancer Genome Atlas (TCGA) normal blood and breast cancer (BRCA) sample datasets were identified. 'Non-unique' SNVs were discovered to be relatively common among a sample of 31 individuals, and SNVs specifically present in single individuals (i.e., private variations) were also common.

\section{Materials and methods}

Basic algorithm. The basic approach is indicated in Fig. 1 and the detailed steps are provided in the supporting online material (SOM) file, 'Stoll 2016 SOM Figure 1, detailed protocol'; all of the SOM files are hosted at http://www.universityseminarassociates.com/Supporting_online_material_for_scholarly_pubs. php). Whole exome sequence (WXS) sample manifest files were downloaded from the Cancer Genomics Hub Browser (browser.cghub.ucsc.edu; approved NIH dbGaP project number 6300) using the following filters: 'Breast invasive carcinoma' under 'By Disease'; 'Blood Derived Normal' and 'Primary Solid Tumor' under 'By Sample Type'; 'WXS' under 'By Library Type'; and 'GRCH37/HG19' under 'By Assembly'. 

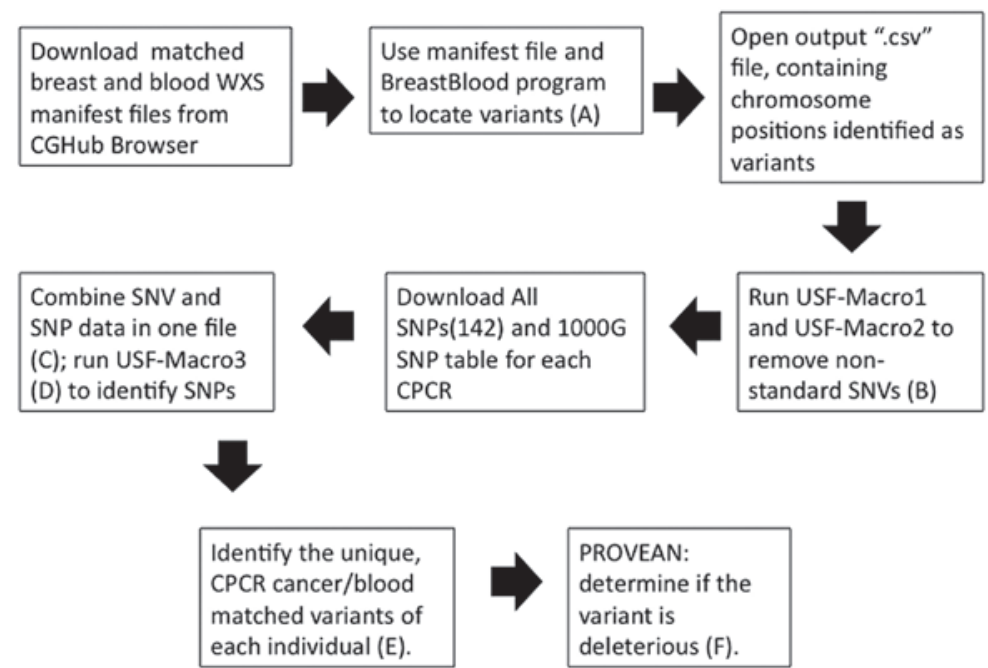

Figure 1. Flow chart representing the processing steps for generating Tables II and III, and Figs. 2 and 3. The upper case letters in parentheses refer to Excel files in the SOM produced by the indicated step in the flow chart. However, (C) in the SOM represents an example file, i.e., SNP removal using the All SNPs(142) database. SOM, supporting online material; WXS, whole exome sequence; SNV, single nucleotide variant; SNP, single nucleotide polymorphism; USF, University of South Florida; CPCR, cytoskeleton-related protein coding regions; PROVEAN, Protein Variation Effect Analyzer.

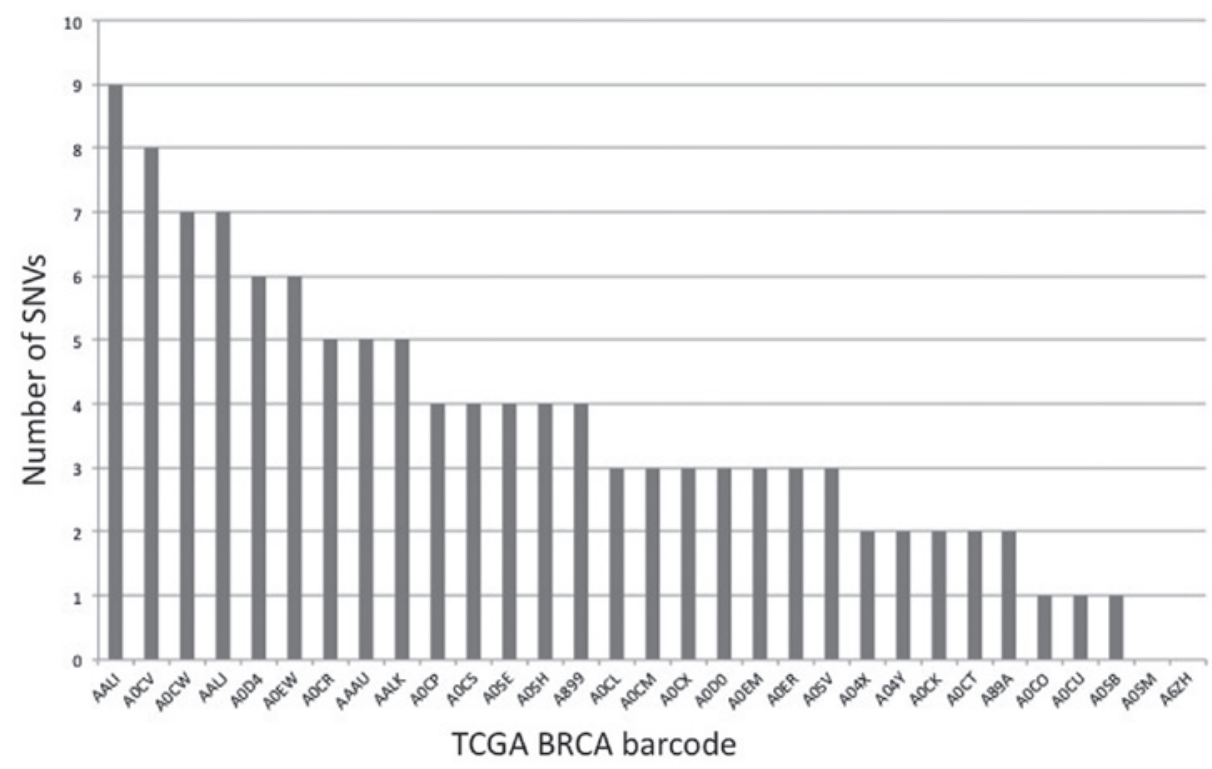

Figure 2. Bar chart representing the number of cancer-/blood-matched CPCR SNVs limited to the indicated, individual barcodes (i.e., that are limited to a single individual). The horizontal axis represents The Cancer Genome Atlas (TCGA) barcodes while the vertical axis represents the number of cancer-/blood-matched CPCR SNVs present represented by the indicated barcode. CPCR, cytoskeletal protein-related coding regions; SNV, single nucleotide variant.

Search results were sorted by 'Barcode' so that solid tumor samples were immediately followed by the matched blood sample. The manifest files for matched BRCA and normal blood samples were downloaded and placed into a file labeled 'Blood' to be consistent with the language of the BreastBlood. sh coding program (Stoll 2016 SOM Figure 1A-BreastBlood) written specifically for the current study. The manifest files were subsequently processed with the BreastBlood.sh to create .csv output files (from the original .xml manifest files). The original programming code, BreastBlood.sh, and output files (Stoll 2016 SOM Figure 1E-BreastBlood Variants) are provided in the SOM. Note that the output files have been further processed using various macros, also provided in the SOM, to eliminate previously identified variants that will not be included in analysis. BreastBlood.sh was designed to identify variants in cytoskeletal protein-related coding regions (CPCRs). The CPCR list was generated from the study by Parry and Blanck (5), representing the most commonly mutated CPCRs in five cancer datasets that were investigated plus two additional CPCRs that are commonly mutated in the melanoma (skin cutaneous melanoma) dataset, which were not evaluated in the previous study (5). The CPCRs are presented in Table I.

Use of SNP databases and the PROVEAN web tool. Two SNP databases [All SNPs(142) and 1000 Genomes; genome.ucsc. edu] were used to filter the previously identified variants in the raw sequence files representing the matched BRCA and 
Table I. HUGO symbols for the cytoskeletal protein-related coding regions set.

\begin{tabular}{|c|c|}
\hline HUGO symbol & Gene name \\
\hline ANK2 & Ankyrin 2 , neuronal \\
\hline APC & Adenomatous polyposis coli \\
\hline COL11A1 & Collagen, type XI, $\alpha 1$ \\
\hline DNAH10 & Dynein, axonemal, heavy chain 10 \\
\hline DNAH11 & Dynein, axonemal, heavy chain 11 \\
\hline DNAH3 & Dynein, axonemal, heavy chain 3 \\
\hline DNAH5 & Dynein, axonemal, heavy chain 5 \\
\hline DNAH7 & Dynein, axonemal, heavy chain 7 \\
\hline DNAH8 & Dynein, axonemal, heavy chain 8 \\
\hline DSCAM & Down syndrome cell adhesion molecule \\
\hline DST & Dystonin \\
\hline FAT3 & FAT atypical cadherin 3 \\
\hline FAT4 & FAT atypical cadherin 4 \\
\hline FBN2 & Fibrillin 2 \\
\hline FGFR1 & Fibroblast growth factor receptor 1 \\
\hline FLG & Filaggrin \\
\hline MUC16 & Mucin 16, cell surface associated \\
\hline MUC17 & Mucin 17 , cell surface associated \\
\hline MUC4 & Mucin 4, cell surface associated \\
\hline NEB & Nebulin \\
\hline NEFH & Neurofilament, heavy polypeptide \\
\hline NF1 & Neurofibromin 1 \\
\hline PCDH15 & Protocadherin-related 15 \\
\hline PCDHAC2 & Protocadherin alpha subfamily $\mathrm{C}, 2$ \\
\hline PCDHGC5 & Protocadherin gamma subfamily $\mathrm{C}, 5$ \\
\hline PCLO & Piccolo presynaptic cytomatrix protein \\
\hline PKHD1 & $\begin{array}{l}\text { Polycystic kidney and hepatic disease } 1 \\
\text { (autosomal recessive) }\end{array}$ \\
\hline PLEC & Plectin \\
\hline RELN & Reelin \\
\hline SPTA1 & Spectrin, alpha, erythrocytic 1 \\
\hline SPTAN1 & Spectrin, alpha, non-erythrocytic 1 \\
\hline SSPO & SCO-spondin \\
\hline SYNE1 & $\begin{array}{l}\text { Spectrin repeat containing, } \\
\text { nuclear envelope } 1\end{array}$ \\
\hline SYNE2 & $\begin{array}{l}\text { Spectrin repeat containing, } \\
\text { nuclear envelope } 2\end{array}$ \\
\hline TTN & Titin \\
\hline XIRP2 & Xin actin binding repeat containing 2 \\
\hline
\end{tabular}

HUGO, Human Genome Organisation.

normal blood barcodes, as indicated in Fig. 1. An example of this process is provided in two SOM files, 'Stoll 2016 SOM Figure 1C (All SNPs(142) Example)' and 'Stoll 2016 SOM Figure 1C (All SNPs(142) Example, Excel)'. Variants not identified by either SNP database were classified by frequency. The classification of variants can be found in SOM Excel file, 'Stoll 2016 SOM Figure 1E-BreastBlood Variants' in sheet 'Variant Color-Coding.' Unique matched variants were
Table II. Deleterious amino acids identified in the cancer-/blood-matched cytoskeletal protein-related coding region single nucleotide variants and limited to one barcode (individual).

\begin{tabular}{|c|c|c|c|c|}
\hline $\begin{array}{l}\text { Residue } \\
\text { (wild-type) }\end{array}$ & $\begin{array}{l}\text { Residue } \\
\text { (mutant) }\end{array}$ & $\begin{array}{c}\text { PROVEAN } \\
\text { prediction }\end{array}$ & $\begin{array}{l}\text { Gene } \\
\text { name }\end{array}$ & $\begin{array}{l}\text { Amino } \\
\text { acid length }\end{array}$ \\
\hline \multicolumn{5}{|c|}{ Barcode: AAAU } \\
\hline A & $\mathrm{V}$ & Deleterious & TTN & $27118^{\mathrm{a}}$ \\
\hline \multicolumn{5}{|c|}{ Barcode: AALI } \\
\hline G & $\mathrm{C}$ & Deleterious & FLG & 4061 \\
\hline $\mathrm{R}$ & $\mathrm{C}$ & Deleterious & DST & 5171 \\
\hline \multicolumn{5}{|c|}{ Barcode: A899 } \\
\hline $\mathrm{P}$ & $\mathrm{L}$ & Deleterious & DNAH7 & 4024 \\
\hline \multicolumn{5}{|c|}{ Barcode: AALJ } \\
\hline $\mathrm{H}$ & $\mathrm{Y}$ & Deleterious & TTN & $27118^{a}$ \\
\hline \multicolumn{5}{|c|}{ Barcode: A0SE } \\
\hline G & $\mathrm{S}$ & Deleterious & MUC4 & 1176 \\
\hline \multicolumn{5}{|c|}{ Barcode: A0CL } \\
\hline $\mathrm{L}$ & $\mathrm{S}$ & Deleterious & FAT3 & 892 \\
\hline \multicolumn{5}{|c|}{ Barcode: A0CP } \\
\hline $\mathrm{P}$ & $\mathrm{T}$ & Deleterious & $\mathrm{TTN}$ & $33423^{a}$ \\
\hline \multicolumn{5}{|c|}{ Barcode: A0CS } \\
\hline $\mathrm{E}$ & $\mathrm{K}$ & Deleterious & TTN & $27118^{\mathrm{a}}$ \\
\hline \multicolumn{5}{|c|}{ Barcode: A0CT } \\
\hline $\mathrm{K}$ & $\mathrm{E}$ & Deleterious & DNAH10 & 4471 \\
\hline \multicolumn{5}{|c|}{ Barcode: A0CV } \\
\hline $\mathrm{C}$ & Y & Deleterious & RELN & 3458 \\
\hline \multicolumn{5}{|c|}{ Barcode: A0CW } \\
\hline $\mathrm{L}$ & $\mathrm{R}$ & Deleterious & MUC16 & 14507 \\
\hline $\mathrm{D}$ & $\mathrm{Y}$ & Deleterious & ANK2 & 3924 \\
\hline \multicolumn{5}{|c|}{ Barcode: A0ER } \\
\hline $\mathrm{R}$ & $\mathrm{C}$ & Deleterious & SYNE2 & 6818 \\
\hline \multicolumn{5}{|c|}{ Barcode: A0EM } \\
\hline V & A & Deleterious & TTN & 27118 \\
\hline \multicolumn{5}{|c|}{ Barcode: AALK } \\
\hline $\mathrm{S}$ & $\mathrm{L}$ & Deleterious & NEB & 6669 \\
\hline
\end{tabular}

${ }^{a}$ Differing lengths represent different protein IDs. PROVEAN, Protein Variation Effect Analyzer.

defined as SNVs that were present in the BRCA and normal blood sample matched barcodes. A histogram of the number of cancer-/blood-matched CPCR SNVs limited to a single individual is presented in Fig. 2. These SNVs, as well as the non-unique SNVs identified among the investigated set of 31 individuals in this report (but not present in the SNP databases) served as input for the Protein Variation Effect Analyzer (PROVEAN) human genome variants program (http://provean.jcvi.org/genome_submit_2.php). This tool was used to determine if the matched mutations caused damaging amino acid changes. A summary of the PROVEAN results is presented in Table II. In addition, the SIFT analysis 
Table III. Amino acid changes identified in the cancer-/blood-matched cytoskeletal protein-related coding region single nucleotide variants of multiple individuals.

\begin{tabular}{lccc}
\hline $\begin{array}{l}\text { Residue } \\
\text { (wild type) }\end{array}$ & $\begin{array}{c}\text { Residue } \\
\text { (mutant) }\end{array}$ & $\begin{array}{c}\text { PROVEAN } \\
\text { prediction }\end{array}$ & $\begin{array}{c}\text { Gene } \\
\text { name }\end{array}$ \\
\hline
\end{tabular}

3,195505859; Barcodes: A0SE, A0CR

$\mathrm{T}$

3, 195507398; Barcodes: A89A, AALJ, A0SB,

A0SE, A0CL, A0CR, A0CO, A0EW, A0CS

D

3, 195507399; Barcodes: A0SB, A0SM,

AALK, A0CV, A0CR, A0CO, A0EW

$\mathrm{T}$

3,195507406; Barcodes: A0CL, AALJ

$\mathrm{P}$

3, 195507422; Barcodes: A89A, A0SE, A0CL, A0CR, A0CO

D

3, 195507443; Barcodes: AAAU, A89A, AALJ, A0SB, A0CL $\mathrm{T}$

3,195507445; Barcodes: A0SB, A0CL, A0CS

$\mathrm{D}$

3,195508667; Barcodes: A89A, AALK, A0CR

$\mathrm{T}$

3,195508670; Barcodes: A89A, AALK, A0CR

D

3,195510238; Barcodes: A0SB, AALK, A0CL

L
A

A

$\mathrm{N}$

Neutral

MUC4

4442

$\mathrm{T}$

Neutral

MUC4

4442

L

Neutral

MUC4

4442

H

Neutral

MUC4

4442

P

Neutral

MUC4

4442

V

Neutral

MUC4

4442

A

Neutral

MUC4

4442

H

Neutral

MUC4

4442

P

Neutral

MUC4

4442

3, 195510310; Barcodes: A89A, A0SB, A0CV, A0CO, A0CR, A0EW

$\mathrm{Y}$

3, 195510341; Barcodes: A89A, A0SB, A0SE, A0CW, A0CV, AALK, A0CO, A0CR, A0EW, A0CS

3,195510610; Barcodes: A0SE, A0CR

$\mathrm{L}$

3, 195510611; Barcodes: A0SB, A0SE

$\mathrm{L}$

3, 195510613; Barcodes: A0SB, A0SE, A0CO

$\mathrm{S}$

3,195510622; Barcodes: A0CW, A0EW

$\mathrm{P}$

1, 103468336; Barcodes: A899, A89A, A6ZH, AALJ, A0CU, A0CT, A0EM, A0CM, A04Y, A04X, A0D0, A0EW

$\mathrm{G}$

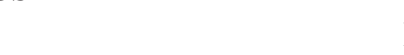

P

$\mathrm{P}$

I

S

Neutral

MUC4

4442

Neutral $\quad$ MUC4 4442

$\mathrm{P}$

Neutra

MUC4

4442

$\mathrm{N}$

Neutral

MUC4

4442

Neutral

MUC4

4442

$\mathrm{H}$

Neutral

MUC4

4442

G

Neutral

PROVEAN, Protein Variation Effect Analyzer.

(http://provean.jcvi.org/genome_submit_2.php) of amino acid substitutions is available at the indicated web site and the results for the current project for the SIFT and PROVEAN analyses are present in the SOM files labeled 'Stoll 2016 SOM Figure 1E-Provean Results, aa variants unique to a barcode' and 'Stoll 2016 SOM Figure 1F-Provean Results, aa variants unique to a barcode'.

Statistical analysis. Statistical significance was determined using Student's t-test with Microsoft Excel (version 2010). 


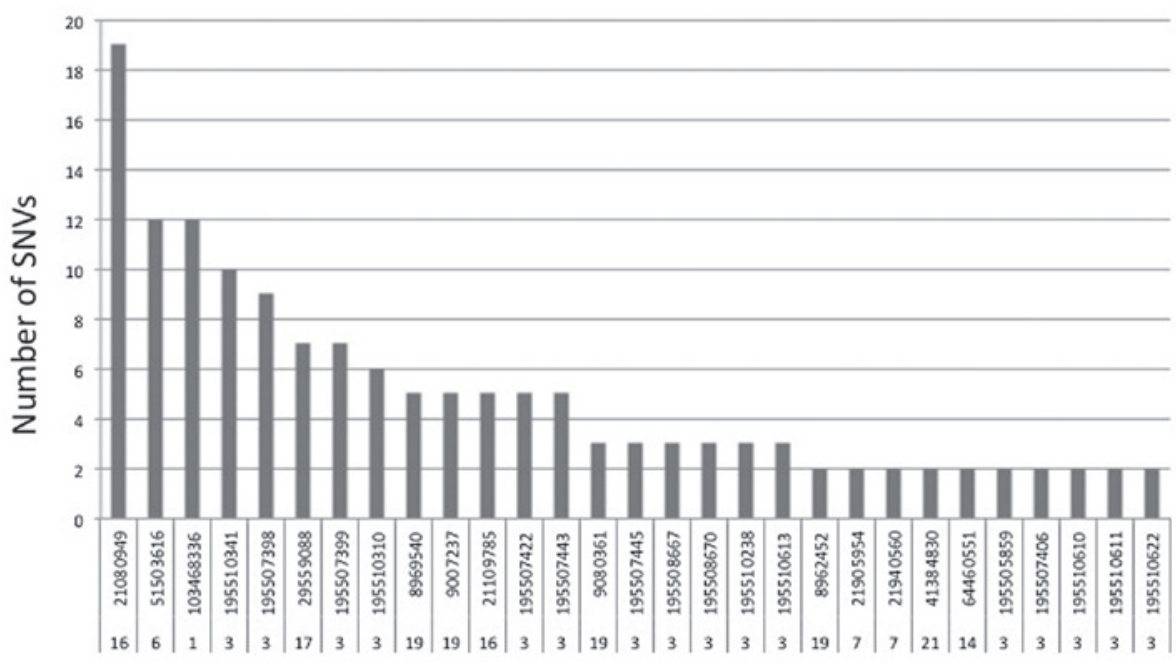

Chromosome and hg19 nucleotide position

Figure 3. Bar chart representing the CPCR SNVs identified in multiple individuals that are not detected by All SNPs(142) or 1000 Genomes databases. The lower number on the horizontal axis indicates the chromosome number, while the upper number indicates the nucleotide position in the hg19 reference genome. The vertical axis represents the number of individuals that possess the indicated SNV. CPCR, cytoskeletal-related protein coding regions; SNV, single nucleotide variant; SNP, single nucleotide polymorphism.

$\mathrm{P}<0.05$ was considered to indicate a statistically significant difference.

\section{Results}

SNVs unique to individuals of the BRCA study set and representing deleterious amino acids. To assess SNVs potentially present in CPCRs (Table I) of BRCA and normal blood samples of a single individual, a series of processing steps, indicated in Fig. 1 were performed. Any SNVs that were present in the All SNPs(142) and 1000 Genome SNP databases (Fig. 1) were eliminated from further consideration. In addition, any SNVs that were not verified by 20 reads or more were eliminated from consideration. Results indicated that 29 barcodes had CPCR SNVs, i.e., nucleotides not matching the reference human genome (Fig. 2). Of these, 14 barcodes represented deleterious amino acid replacements, according to PROVEAN analysis, in the following coding regions: TTN, MUC4, FLG, DST, DNAH7, FAT3, DNAH10, RELN, MUC16, ANK2, SYNE2 and NEB (Table II). The SIFT analysis indicated 21 damaging mutations (SOM Excel file labeled, 'Stoll 2016 SOM Figure 1F-Provean Results, aa variants unique to a barcode').

SNVs shared among members of the BRCA study set, but not present in common SNP databases. The results of the current SNV analyses indicated that a number cancer-/blood-matched CPCR SNVs were shared between barcodes in this analysis group of 31 barcodes, despite using the above two SNP databases to clear the known SNPs. Fig. 3 demonstrates the chromosome and nucleotide positions of the cancer-/blood-matched SNVs identified in more than one barcode, as well as the number of barcodes with a given variant. These SNVs were also analyzed using the PROVEAN tool (Table III; Stoll 2016 SOM Figure 1G-Provean Results, aa variants present in more than one barcode). A summary of the specific, non-unique CPCR
SNVs is provided in Table III. Thus, 112 SNVs unique to one individual (Fig. 2) indicated 16 deleterious AA substitutions (Table II), whereas 29 SNVs shared among the barcodes represented zero deleterious AA substitutions, using the PROVEAN analysis $(\mathrm{P}<0.00002$; Student's t-test). The SIFT analysis detected 21 deleterious AA among the unique, matched SNVs, and there were three deleterious AA substitutions among the shared, matched SNVs. The SIFT analysis maintains the trend indicated by the PROVEAN analysis, but was not statistically significant.

\section{Discussion}

The above data and analyses indicate that systemic mutations in CPCRs are relatively common, consistent with the large size of these coding regions. In general, systemic, de novo mutations are considered sporadic and unpredictable, as in the case of Rett's syndrome, which can be caused by a point mutation in methyl $\mathrm{CpG}$ binding protein-2 (6). However, the large size of the CPCRs renders it more likely that de novo, or relatively recently generated SNVs, will be present in the CPCRs. Furthermore, the above analyses indicate that SNVs that lead to deleterious amino acid substitutions are more common when not shared.

The relatively common occurrences of these de novo, systemic deleterious amino acid substitutions in CPCRs raises questions regarding the value of evaluating these coding regions. For example, small coding regions are unlikely to have de novo mutations and, thus, there may not be a practical justification for routine analysis. However, if de novo CPCR SNVs are identified to be essentially inevitable, investigating them would be valuable, particularly with regard to potentially identifying deleterious amino acid alternations. Such routine analysis may have value in better understanding a range of medical conditions, for example heart disease, due to the major role of various CPCRs in the formation of the sarcomere cytoskeleton (7). 
In addition, the results cause us to query other coding region groups, where individual group members may be too small to regularly reveal systemic mutations. The entire group collection, however, may be large enough such that de novo systemic mutations would alter one member of the group often enough to justify screening. For example, a comprehensive collection of tumor suppressor genes would likely have a member that represents a systemic mutation, due to random chance, fairly often. While such a collection is not likely to be as large as a CPCR collection, it is likely a large enough portion of genome, with mutations affecting a large enough number of individuals, to justify screening. Furthermore, due to the degeneracy of numerous tumor suppressor signaling pathways (8), a mutation in any one tumor suppressor, among a set, may represent pre-disposition to cancer.

In conclusion, SNPs not present in the SNP databases used here, but that were present in more than one of the individuals investigated above, and potentially present in other SNP databases, were detected. However, as all of the individuals analyzed in the current study were BRCA patients, the outcome of multiple shared SNVs results in the hypothesis that certain de novo, systemic CPCR mutations may facilitate BRCA development. Future studies are required to address the occurrence of potential, cancer specific SNVs in the CPCR set by repeating the above analyses for multiple types of cancer.

\section{Acknowledgements}

Authors would like to acknowledge support from the Anna Valentine program and the University of South Florida research computing facility, in particular, Dr Tony Green.

\section{References}

1. Parry ML, Ramsamooj M and Blanck G: Big genes are big mutagen targets: A connection to cancerous, spherical cells? Cancer Lett 356 (2 Pt B): 479-482, 2015.

2. Narsing S, Jelsovsky Z, Mbah A and Blanck G: Genes that contribute to cancer fusion genes are large and evolutionarily conserved. Cancer Genet Cytogenet 191: 78-84, 2009.

3. Pava LM, Morton DT, Chen R and Blanck G: Unifying the genomics-based classes of cancer fusion gene partners: Large cancer fusion genes are evolutionarily conserved. Cancer Genomics Proteomics 9: 389-395, 2012.

4. Fawcett TJ, Parry ML and Blanck G: A Novel Approach to Evaluating Cancer Driver Gene Mutation Densities: Cytoskeleton-related Gene Candidates. Cancer Genomics Proteomics 12: 283-290, 2015.

5. Parry ML and Blanck G: Flat cells come full sphere: Are mutant cytoskeletal-related proteins oncoprotein-monsters or useful immunogens? Hum Vaccin Immunother 12: 120-123, 2016.

6. Ballestar E, Yusufzai TM and Wolffe AP: Effects of Rett syndrome mutations of the methyl-CpG binding domain of the transcriptional repressor $\mathrm{MeCP} 2$ on selectivity for association with methylated DNA. Biochemistry 39: 7100-7106, 2000.

7. Kontrogianni-Konstantopoulos A, Ackermann MA, Bowman AL, Yap SV and Bloch RJ: Muscle giants: Molecular scaffolds in sarcomerogenesis. Physiol Rev 89: 1217-1267, 2009.

8. Ford SA and Blanck G: Signal persistence and amplification in cancer development and possible, related opportunities for novel therapies. Biochim Biophys Acta 1855: 18-23, 2014. 